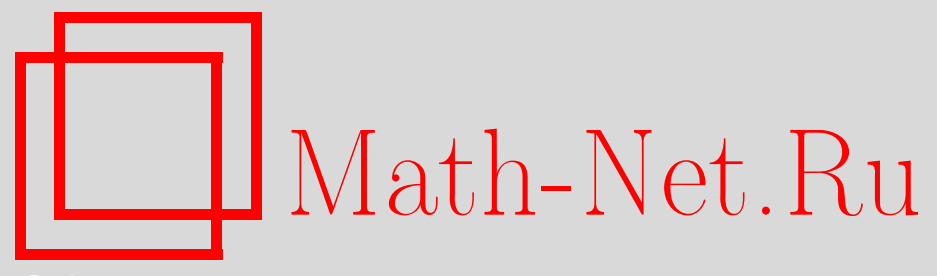

И. А. Семаев, О вычислении логарифмов на эллиптических кривых, Дискрет. матем., 1996, том 8, выпуск $1,65-71$

DOI: https://doi.org/10.4213/dm516

Использование Общероссийского математического портала Math-Net.Ru подразумевает, что вы прочитали и согласны с пользовательским соглашением http://www . mathnet.ru/rus/agreement

Параметры загрузки:

IP : 34.229 .108 .108

26 апреля 2023 г., $12: 35: 43$

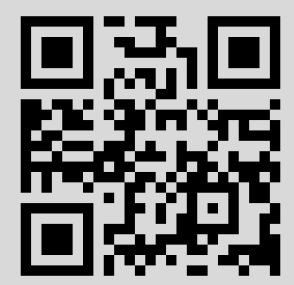


УДК 519.7

\title{
О вычислении логарифмов на эллиптических кривых
}

\author{
(C) 1996 г. И. А. Семаев
}

\begin{abstract}
Рассматривается задача решения показательного уравнения в циклической подгруппе порядка $m$ группы $E$ точек эллиптической кривой, определенной над конечным полем $F_{q}$. Доказано, что если $F_{q_{1}}$ - минимальное расширение $F_{q}$ такое, что подгруппа точек, рациональных над $F_{q_{1}}$, группы $E$ содержит подгруппу, изоморфную $\mathbf{Z} / m \times \mathbf{Z} / m$, то сложность решения указанного уравнения не превосходит сложности вычисления логарифмов в поле $F_{q_{1}}$ или сложности $O(\ln m)$ арифметических операций в әтом поле. Таким образом, вычисление логарифмов на әллиптических кривых сводится к логарифмированию в конечном поле. С помощью другого подхода эта редукция была получена Менезесом, Окамото и Ванстоуном.
\end{abstract}

1. Рассматривается задача решения показательного уравнения в группе точек эллиптической кривой, определенной над конечным полем $F_{q}$. Поле $F_{q}$ состоит из $q$ әлементов. Пусть $F$ - некоторое расширение исходного поля $F_{q}$. Точки $(x, y), x, y \in F$, удовлетворяющие уравнению

$$
y^{2}+a_{1} x y+a_{3} y=x^{3}+a_{2} x^{2}+a_{4} x+a_{6},
$$

где $a_{i} \in F_{q}$ таковы, что кривая (1) без особенностей, вместе с бесконечноудаленной точкой $P_{\infty}$ образуют коммутативную группу, которую мы будем обозначать $E / F$. Известно, что если $F$ - конечное поле порядка $q_{1}$, то порядок $N_{q_{1}}$ группы $E / F$ удовлетворяет неравенству $\left|N_{q_{1}}-q_{1}-1\right| \leqslant 2 q_{1}^{1 / 2}$.

Пусть $T$ - некоторая точка на $E / F_{q}$, которая порождает циклическую подгруппу $\langle T\rangle$ порядка $m$. Таким образом, $|\langle T\rangle|=m$. Задача состоит в решении уравнения

$$
S=n T
$$

относительно $n$ при известных $S$ и $T$. Нетрудно понять, что решение уравнения (2) сводится к случаю, когда $m-$ простое число.

Кроме тривиального перебора $n$ от 1 до $m$ с последующей проверкой выполнения равенства (2), существует метод, предложенный Полигом и Хеллманом [1]. При памяти объема $V$ сложность решения уравнения (2) есть величина порядка $m / V+V$. Сравним сложность решения уравнения (2) со сложностью проблемы дискретного логарифмирования. Рассмотрим уравнение

$$
b=a^{n},
$$


$a, b \in F_{q}^{*}$. Проблема решения уравнения (3) имеет обширную литературу. Приведем два наиболее ярких результата. Пусть $q \rightarrow \infty$. В работе [2] Адлеман построил алгоритм в простом поле, асимптотическая оценка сложности которого равна

$$
\exp \left(c_{1}(\ln q \ln \ln q)^{1 / 2}\right)
$$

Копперсмит в [3] установил, что в случае, когда $q=2^{k}$, сложность логарифмирования равна величине

$$
\exp \left(c_{2}\left(k \ln ^{2} k\right)^{1 / 3}\right)
$$

В том и другом случае $c_{1}$ и $c_{2}$ - небольшие постоянные. Следует также отметить, что доказательства оценок (4) и (5) имеют эвристический характер.

Как видно, проблема дискретного логарифмирования является во многих случаях существенно менее сложной по сравнению с проблемой решения уравнения (2). Отсутствие быстрых алгоритмов решения уравнения (2) является основанием для использования этой задачи в области криптографии при построении сетей связи с открытым распределением ключей $[4,7]$.

В предлагаемой работе доказывается, что с помощью несложных вычислений решение уравнения (2) сводится к решению уравнения (3) в некотором расширении $F_{q_{1}}$ исходного поля $F_{q}$. Примеры показывают, что степень указанного расширения может быть очень мала. Это приводит к резкому уменьшению сложности вычисления логарифмов на эллиптических кривых, обладающих таким свойством. Точнее, в работе доказывается следуюшая теорема.

Теорема 1. Пусть $F_{q_{1}}$ - расширение поля $F_{q}$ такое, что $E / F_{q_{1}}$ содержст подгруппу, изоморфную $\mathbf{Z} / m \times \mathbf{Z} / m$. Тогда сложсность решения уравнения (2) не превосходит слохсности вычисления логарифмов в поле $F_{q_{1}}$ или слохсности $O(\ln m)$ арифметических операчий в том жсе поле.

Статья, кроме введения, содержит еще два раздела. В разделе 2 вводятся некоторые понятия и приводятся отдельные результаты теории эллиптических кривых. В разделе 3 доказывается сформулированная теорема и обсуждаются примеры.

Предлагаемая работа была выполнена в 1990 году. Другим методом этот результат получен в [10], некоторые усовершенствования и обобщения содержатся в [11]. Следует отметить, что методы автора могут быть применены к решению показательного уравнения в произвольном абелевом многообразии, определенном над конечным полем, при подходящем задании абелева многообразия.

2. Основное содержание этого параграфа взято из книги [5].

Пусть $F$ - алгебраическое замыкание поля $F_{q}$, а $F[x, y]$ - кольцо многочленов от двух переменных $x, y$. Поле частных $F(E)$ фактор-кольца $F[x, y]$ по идеалу, порождаемому уравнением (1), называется полем алгебраических функций на кривой $E / F$. Дивизором на $E / F$ называется формальная сумма конечного числа точек кривой с целыми коэффициентами. Таким образом, дивизор на $E / F$ имеет вид $\sum n_{P}(P)$, где среди целых $n_{P}$ лишь конечное число отлично от нуля. Пусть $f \in F(E)$ - функция на $E / F$. Точки $P_{i}$ - нули функции $f$ с кратностями 
$n_{i}$, а $Q_{j}$ - полюса функции $f$ с кратностями $m_{j}$. Тогда дивизором функции $f$ называется дивизор $\sum n_{i}\left(P_{i}\right)-\sum m_{j}\left(Q_{j}\right)$, который обозначается $\operatorname{div} f$. Пусть

$$
D=\sum n_{P}(P)
$$

- произвольный дивизор на $E / F$. Известно, что существует алгебраическая функция $f \in F(E)$ такая, что $\operatorname{div} f=D$ тогда и только тогда, когда одновременно выполняются равенства $\sum n_{P}=0$, как сумма целых чисел, и $\sum n_{P}(P)=P_{\infty}$, в группе точек эллиптической кривой $E / F$. Можно определить значение функции $f$ на дивизоре $D$, если нули и полюса $f$ не содержатся среди точек $P$ таких, что $n_{P} \neq 0$ в выражении (6). Положим

$$
f(D)=\prod_{P} f^{n_{P}}(P) .
$$

Пусть $F_{q_{1}}$ - расширение поля $F_{q}$ такое, что группа $E / F_{q_{1}}$ содержит подгруппу $E[m]$, изоморфную $\mathbf{Z} / m \times \mathbf{Z} / m$. Спаривание Вейля $e_{m}$ - билинейное отображение прямого произведения $E[m]$ на себя в мультипликативную группу поля $F_{q_{1}}: e_{m}: E[m] \times E[m] \rightarrow F_{q_{1}}^{*}$. Для пары точек $P, Q \in E[m]$ значение функции $e_{m}(P, Q)$ определяется следующим образом. Пусть $R$ - любая точка на $E / F$, обладающая тем свойством, что точки $P_{\infty}, P, Q+R, R$ попарно различны. Определим дивизоры на $E / F$, полагая $D_{P}=(P)-\left(P_{\infty}\right), D_{Q}=(Q+R)-(R)$. Тогда найдутся алгебраические функции $f_{P}, f_{Q} \in F(E)$ с коэффициентами из поля $F q_{1}$, удовлетворяющие равенствам

$$
\begin{aligned}
& \operatorname{div} f_{P}=m(P)-m\left(P_{\infty}\right), \\
& \operatorname{div} f_{Q}=m(Q+R)-m(R) .
\end{aligned}
$$

Теперь значение спаривания Вейля точек $P$ и $Q$ определяется равенством $e_{m}(P, Q)=f_{P}\left(D_{Q}\right) / f_{Q}\left(D_{P}\right)$. Заметим, что функции, которые удовлетворяют равенствами (7) и (8), определены с точностью до мультипликативных постоянных из $F$. Очевидно, что значение функции $e_{m}$ не зависит от этих множителей. Функция $e_{m}$ обладает следующими свойствами:

(a) $e_{m}\left(P_{1}+P_{2}, Q\right)=e_{m}\left(P_{1}, Q\right) e_{m}\left(P_{2}, Q\right)$;

(b) $e_{m}(P, Q)=e_{m}(Q, P)^{-1}$;

(c) если $\sigma$ - автоморфизм $F$ над $F_{q}$, то $e_{m}(P, Q)^{\sigma}=e_{m}\left(P^{\sigma}, Q^{\sigma}\right)$;

(d) существуют точки $P, Q \in E[m]$ такие, что $e_{m}(P, Q)$ - примитивный корень степени $m$ из 1.

Из свойства (d) следует, в частности, что мультипликативная группа поля $F_{q_{1}}$ содержит всю группу корней степени $m$ из 1 .

Теперь ясно, каким образом свести решение уравнения (2) к решению уравнения (3). Пусть $Q$ - произвольная точка $E[m]$ порядка $m$ такая, что группы $\langle Q\rangle$ и $\langle T\rangle$ имеют тривиальное пересечение. Тогда из свойств спаривания Вейля следует, что отображение $e_{m}(\cdot, Q)$ осуществляет изоморфное вложение группы $\langle T\rangle$ в группу $F / F_{q_{1}}^{*}$. Поэтому имеет место соотношение

$$
e_{m}(S, Q)=e_{m}(T, Q)^{n}
$$


в поле $F_{q_{1}}$. Таким образом, для доказательства теоремы осталось показать, что значения $e_{m}(S, Q)$ и $e_{m}(T, Q)$ могут быть вычислены за $O(\ln m)$ арифметических операций в поле $F_{q_{1}}$. Следует отметить, что точка $Q$ может быть легко вычислена путем случайного выбора точек в группе $E / F_{q_{1}}$. Эта операция является единственным вероятностным моментом в предлагаемой процедуре.

3. Обозначим через $f_{Q}^{\prime}$ алгебраическую функцию на $E / F$ с дивизором $\operatorname{div} f_{Q}^{\prime}=$ $m(Q)-m\left(P_{\infty}\right)$. Очевидно, что $f_{Q}^{\prime}(X)=f_{Q}(X-R)$. Поэтому, учитывая выражение (9) для $e_{m}$, а также рассуждение в конце предыдущего параграфа, для доказательства теоремы достаточно установить справедливость следующей леммы.

Лемма 1. Пусть $P \in E[m]$, точка $U-$ произвольная точка жа $E / F$, которая не содержится в группе $\langle P\rangle$, порождаемой точкой $P$. Тогда значение функции $f_{P}$, которая определена равенством (7), в точке $U$ мохсет быть вычислено за $O(\ln m)$ арифметических операчий в поле $F_{q_{1}}$.

Доказательство. В поле $F(E)$ существует и определена над $F_{q_{1}}$ алгебраическая функция $\varphi_{k}$, которая удовлетворяет равенству

$$
\operatorname{div} \varphi_{k}=k(P)-(k P)-(k-1)\left(P_{\infty}\right) .
$$

В частном случае, когда $k=m$, имеют место равенства

$$
\operatorname{div} \varphi_{m}=m(P)-(m P)-(m-1)\left(P_{\infty}\right)=m(P)-m\left(P_{\infty}\right)=\operatorname{div} f_{P} .
$$

Поэтому с точностью до множителя из $F$

$$
\varphi_{m}=f_{P}
$$

Мы покажем, как быстро вычислить значение функции $\varphi_{m}$ в точке $U$. Тогда из (10) получим утверждение леммы. Предположим, что $k=k_{1}+k_{2}$. Имеет место тождество

$$
\varphi_{k}=\lambda_{k_{1}, k_{2}}^{-1} \varphi_{k_{1}} \varphi_{k_{2}}
$$

где $\lambda_{k_{1}, k_{2}}$ - функция на $E / F$, дивизор которой равен

$$
(k P)-\left(k_{1} P\right)-\left(k_{2} P\right)+\left(P_{\infty}\right) .
$$

Тождество (11) есть следствие цепочки равенств

$$
\begin{aligned}
\operatorname{div} \varphi_{k}= & k(P)-(k P)-(k-1)\left(P_{\infty}\right) \\
= & k_{1}(P)-\left(k_{1} P\right)-\left(k_{1}-1\right)\left(P_{\infty}\right)+k_{2}(P)-\left(k_{2} P\right)-\left(k_{2}-1\right)\left(P_{\infty}\right) \\
& \quad-\left((k P)-\left(k_{1} P\right)-\left(k_{2} P\right)+\left(P_{\infty}\right)\right) \\
= & \operatorname{div} \varphi_{k_{1}}+\operatorname{div} \varphi_{k_{2}}-\operatorname{div} \lambda_{k_{1}, k_{2}}
\end{aligned}
$$

и того факта, что дивизор произведения функций равен сумме дивизоров этих функций.

Из тождества (11) и 2-адического представления числа $k$ следует, что функция $\varphi_{k}$ является произведением $O(\ln k)$ функций вида $\lambda_{k_{1}, k_{2}}$ и такого же числа функций $\varphi_{2}$. Снова применяя тождество (11), находим, что

$$
\varphi_{2^{l}}=\lambda_{2^{l-1}, 2^{l-1}}^{-1} \varphi_{2^{l-1}}^{2} .
$$


Значит, для того, чтобы получить значения функций $\varphi_{2}$ достаточно вычислить значения функций $\lambda_{2^{l}, 2^{l}}, l=1,2, \ldots,\left[\log _{2} k\right]$. Итак, для вычисления значения алгебраической функции $\varphi_{k}$ надо получить значения $O(\ln k)$ функций, дивизор которых имеет вид (12). Затем следует их перемножить в соответствии с правилами, задаваемыми тождествами вида (11).

Каким образом можно получить значение функции $\lambda_{k_{1}, k_{2}}$ ? Рассмотрим несколько более общую функцию $\lambda$, дивизор которой удовлетворяет равенству $\operatorname{div} \lambda=(R+H)-(R)-(H)+\left(P_{\infty}\right)$, где $R, H$ - произвольные точки на $E / F_{q_{1}}$. Пусть $\eta_{1}, \eta_{2}, \eta_{3}$ - линейные функции с коэффициентами в поле $F_{q_{1}}$, которые задают уравнения прямых, проходящих через точки $R+H,-R,-H$, точки $R$, $-R$, точки $H,-H$, соответственно. Тогда

$$
\begin{aligned}
\operatorname{div} \eta_{1} & =(R+H)+(-R)+(-H)-3\left(P_{\infty}\right) \\
\operatorname{div} \eta_{2} & =(R)+(-R)-2\left(P_{\infty}\right), \\
\operatorname{div} \eta_{3} & =(H)+(-H)-2\left(P_{\infty}\right) .
\end{aligned}
$$

Поэтому $\operatorname{div}\left(\eta_{1} \eta_{2}^{-1} \eta_{3}^{-1}\right)=\operatorname{div} \lambda$, то есть следует положить $\lambda=\eta_{1} \eta_{2}^{-1} \eta_{3}^{-1}$. Отметим, что все алгебраические функции вида $\lambda_{k, l}$ определены над $F_{q_{1}}$. Поэтому вычисление их значений в точке $U \in E / F_{q_{1}}$ производится также над $F_{q_{1}}$. Далее, так как точка $U$ не входит в группу, порождаемую точкой $P$, она не содержится среди нулей и полюсов ни одной из функций $\lambda_{k, l}$. Следовательно, их значения в точке $U$ корректно определены и отличны от нуля. Таким образом, мы показали, что значение алгебраической функции $\varphi_{k}$ в точке $U$ можно вычислить за $O(\ln k)$ операций в поле $F_{q_{1}}$. Полагая $k=m$, получаем утверждение леммы.

Теорема является следствием леммы и рассуждений, приведенных в конце 2 .

Рассмотрим теперь какова степень $k$ расширения поля $F_{q_{1}}$ над $F_{q}$ такого, что $E[m] \in E / F_{q_{1}}$. Докажем еще одну лемму.

Лемма 2. Предположим, что $m$ делит порядок группь $E / F_{q}$. Пусть $l$ - минимальное натуральное число, для которого $\left.q^{l} \equiv 1(\bmod m)\right)$. Тогда степень расширения $k$ делится на $\mathrm{l}$ и делит $\mathrm{lm}$.

Доказательство. Функция $e_{m}$ принимает свои значения в поле $F_{q^{l}}$. Поэтому, очевидно, $l$ делит $k$. Пусть $P$ и $Q$ - точки порядка $m$ на $E / F$. Пусть при этом $P \in E / F_{q}$. Тогда согласно свойству (c) спаривания Вейля

$$
e_{m}(P, Q)=e_{m}(P, Q)^{\sigma}=e_{m}\left(P^{\sigma}, Q^{\sigma}\right)=e_{m}\left(P, Q^{\sigma}\right)
$$

где $\sigma$ - произвольный автоморфизм поля $F$ над $F_{q^{\iota}}$. Отображение $e_{m}(P, \cdot)$, когда порядок точки $P$ равен $m$, осуществляет изоморфное вложение группы $\langle Q\rangle$ в мультипликативную группу поля $F_{q^{l}}$. Поэтому для каждого автоморфизма $\sigma$ поля $F$ над $F_{q^{l}}$ выполняется равенство $Q^{\sigma}=Q+s P$ при некотором $s$. Значит, автоморфизм $\sigma^{m}$ фиксирует точку $Q$. Следовательно, точка $Q$ лежит на $E / F_{q^{l m}}$. Таким образом, число $l$ делит $k$ и число $k$, в свою очередь, делит произведение lm. Лемма доказана.

Рассмотрим примеры. В статье [7] предлагается использовать на практике одну из кривых с комплексным умножением. А именно, пусть $p$ - простое число, 
$p \equiv-1(\bmod 4)$. Эллиптическая кривая имеет над $F_{p}$ уравнение

$$
y^{2}=x^{3}+A x
$$

Известно, что порядок группы $E / F_{p}$ равен $p+1$. Нетрудно показать, что $E[p+1] \subseteq E / F_{p^{2}}$. Поэтому сложность логарифмирования на кривой (13) не превосходит сложности логарифмирования в поле $F_{p^{2}}$. Как показано в [6], для сложности логарифмирования в $F_{p^{2}}$ имеет место оценка (4).

Похожим образом можно показать, что решение показательного уравнения на кривых $y^{2}=x^{3}+B$ над $F_{p}$, где $p \equiv-1(\bmod 3)$ и

$$
y^{2}+y+A=x^{3}
$$

над $F_{2^{k}}$, где $k$ - нечетное число, также сводится к логарифмированию в квадратичном расширении исходного поля. Причем в последнем случае для сложности логарифмирования на кривой (14) верна оценка (5). Эти кривые были рассмотрены с точки зрения практического приложения в работе [8].

Кроме того, в статье [9] предлагается использовать группы точек эллиптических кривых над полем характеристики 2, у которых $j$-инвариант равен 0 . Это кривая (14) при $\mathrm{A}=0$, а также кривые $y^{2}+y=x^{3}+x$ and $y^{2}+y=x^{3}+x+1$. Приведены порядки соответствующих групп точек над полем $F_{2^{k}}$. Нетрудно показать, что логарифмирование в этих группах сводится к логарифмированию в поле $F_{2^{4 k}}$. Значит, для этой задачи верна субъэкспоненциальная оценка (5).

Таким образом, все известные в настоящее время варианты эллиптических кривых, предлагаемые для открытой криптографии, обладают нехорошим свойством. А именно, сложность решения задачи логарифмирования в этих группах не превосходит сложности вычисления логарифма в конечных полях, которые являются расширениями очень небольшой степени исходного поля. Для последней задачи, как указывалось во введении, разработаны эффективные алгоритмы.

\section{Список литературы}

1. Pohlig S., Hellman M. An improved algorithm for computing logarithms over GF(p) and its cryptographic significance. IEEE Trans. Inform. Theory (1978) 24, 106-110.

2. Adleman L. A subexponential algorithm for the discrete logarithm problem with applications to cryptography. In: Proc. IEEE 20th Annual Symp. Foundation Computer Sci. 1979, pp. 55-60.

3. Coppersmith D. Fast evaluation of logarithms in fields of characteristic two. IEEE Trans. Inform. Theory (1984) 30, 587-594.

4. Koblitz N. Elliptic curves cryptosystems. Math. Comput. (1987) 48, 203-209.

5. Silverman J. H. The Arithmetic of Elliptic Curves. Springer, Berlin, 1986.

6. El Gamal T. A subexponential-time algorithm for computing discrete logarithms over GF $\left(p^{2}\right)$. In: Proc. Crypto'83. 1984, pp. 275-292.

7. Miller V. S. Use of elliptic curves in cryptography. Lect. Notes Comput. Sci. (1986) 218, 417-426. 
8. Bender A., Castagnoli G. The implementation of elliptic curve cryptosystems. Lect. Notes Comput. Sci. (1990) 435, 417-426.

9. Menezes A., Vanstone S. The implementation of elliptic curve cryptosystems. Lect. Notes Comput. Sci. (1990) 453, 2-13.

10. Menezes A., Okamoto T., Vanstone S. Reducing elliptic curve logarithms to logarithms in a finite field. In: Proc. 22nd Annual AMC Symp. Theory of Computing. 1991, pp. 80-89.

11. Frey G., Ruck H. G. A remark concerning $m$-divisibility and the discrete logarithm in the divisor class group of curves. Math. Comput. (1994) 62, 865-874.

Статья поступила 23.11.1992. 